\title{
The effect of stimulus current pulse width on nerve fiber size recruitment patterns
}

\author{
Robert B. Szlavik a , Hubert de Bruin a,b, \\ ${ }^{a}$ Department of Electrical and Computer Engineering, McMaster University, Hamilton, Ontario, Canada \\ ${ }^{\mathrm{b}}$ Department of Medicine, McMaster University, Hamilton, Ontario, Canada
}

\begin{abstract}
There have been theoretical studies presented that postulate a change in the stimulus current amplitude required to recruit nerve fibers with different stimulus current pulse widths. Based on these theoretical predictions, it has been suggested that the stimulus pulse width parameter may be used to selectively recruit fibers of different sizes and that this selectivity should increase with increasing distance from the stimulus electrode. In this paper, a simulation study of the recruitment patterns of a population of motor nerve fibers with a histologically accurate fiber diameter distribution is presented. Nerve fiber excitation simulations coupled with a time varying field simulation suggest that, for surface stimulation, there is only a marginal selectivity achievable in the average nerve fiber diameter that is recruited across the range of commonly used stimulus pulse widths but this selectivity also increases with increased electrode distance. Experimental evidence consisting of estimates of nerve fiber diameter based on motor unit latency studies is also presented that is consistent with the predictions made by the electromagnetic field and nerve fiber excitation simulations.
\end{abstract}

Keywords: Nerve stimulation; Volume conductor fields; Stimulus pulse width; Nerve fiber size

\section{Introduction}

The ability to selectively recruit specific nerve fibers in a nerve trunk has been the subject of a large body of research in the area of functional electrical stimulation. Selective recruitment of motor nerve fibers would facilitate greater control in the recruitment of individual motor units for individuals who suffer from central or peripheral nervous system injuries with consequent loss of muscle function. As well, by employing stimulus strategies which continually change or alternate pulse widths, a greater population of motor units could be recruited in a pseudo-asynchronous fashion thus avoiding early fatigue at sub-maximal contractions. In the field of diagnostic electromyography, the ability to selectively recruit motor nerve fibers of different diameters would greatly aid in stimulating, at sub-maximal levels, different populations of motor units or sensory nerves. Several motor unit number estimation techniques, such as that presented by McComas and colleagues rely on stimulating a small sample of motor units $[1,2]$. Such techniques would also become more reliable if the sample nerve fibers or motor units stimulated were more representative of the muscle motor unit population.

The electrical properties of nerve fibers, in relation to their size, has been of interest to neurophysiologists since Erlanger and Gasser's pioneering work on the compound nature of the action potential and their discovery of the relationship between the action potential conduction velocity and nerve fiber size [3]. More recent nerve fiber recruitment selectivity studies which varied stimulus current waveform parameters, have shown that narrower stimulus pulse widths increase the difference between nerve fiber thresholds [4]. These experiments employed direct stimulation where a metal loop and nerve cuff electrodes were placed around the nerve. Several chronically implanted electrode designs have been proposed in the literature with the goal of nerve fascicle and spatial recruitment selectivity [4-6].

Grill and Mortimer's theoretical study, that emphas- 
ized the stimulus strength versus duration effect and placed the nerve fiber in an idealized infinite, isotropic and homogeneous tissue environment, suggested that varying the stimulus current pulse width would be effective in achieving selective recruitment of motor units based on the different electrical recruitment properties for myelinated nerve fibers of varying size [7]. Their simulations demonstrated a greater than fourfold increase in the difference in stimulus current amplitude requirement between $10 \mu \mathrm{m}$ and $20 \mu \mathrm{m}$ fibers at an electrode to fiber distance of $2 \mathrm{~mm}$ for a decrease in stimulus pulse width of $500 \mu \mathrm{s}$ to $10 \mu \mathrm{s}$. At $1 \mathrm{~mm}$, the same change in stimulus pulse duration resulted in an approximate tripling in the difference between the stimulus current amplitude threshold requirement. They further demonstrated spatial selectivity experimentally by showing that shorter stimulus pulse widths allowed for the activation of more fibers localized in a single fascicle over a greater range of stimulus pulse amplitudes before spillover occurred to a fascicle innervating an antagonist muscle group [8]. Although, we have concentrated on stimulus pulse width effects, fiber selectivity can also be achieved by anodal or high frequency blocking [9].

In this study, we utilize surface stimulus and an electromagnetic field simulation coupled with a nerve fiber excitation simulation that can quantitatively predict the nerve fiber recruitment changes resulting from variations in the stimulus current pulse width from $10 \mu$ s to 1000 $\mu$ s. Experimental studies were also conducted to obtain estimates of the nerve fiber diameters of stimulated motor units. These estimates were based on the conduction latencies obtained from motor unit action potentials extracted from the sub-maximal $\mathrm{M}$-wave using a motor unit number estimation technique [2]. These experimental studies were carried out using surface electrode stimulation of the median nerve and varying stimulus pulse widths from $50 \mu$ s to $500 \mu$ s.

The range of stimulus pulse widths used were chosen to investigate whether the simulation results could be confirmed experimentally. Equipment limitations precluded empirical investigation of extremely narrow pulse widths shorter than $50 \mu$ s because current levels would be required that exceeded the legal and equipment limitations imposed on human investigations.

\section{Methodology}

\subsection{Simulation studies of nerve fiber recruitment patterns}

Simulation of the nerve fiber recruitment patterns was a two step process. The first step involved solving for the potential distribution in a rectangular simulation domain with the boundary conditions of zero normal current at the top surface, except for the stimulus site, and zero potential along the other surfaces as per Fig. 1. A monopolar surface stimulus electrode configuration was used for this part of the simulation. As has been the practice of other authors, a purely resistive tissue medium was assumed. However, tissue anisotropy was accounted for in the model using values that are typically quoted for skeletal muscle tissue [10,11] and a surface boundary condition was implemented in order to simulate a more realistic tissue environment. An even more realistic inhomogeneous tissue environment including skin, subcutaneous fat, nerve fascia and fibers was beyond the scope of this paper and are not typically incorporated in nerve excitation models $[12,13]$. As well, the nerve excitations simulations previously mentioned routinely assume a homogeneous, isotropic and infinite medium external to the nerve fiber and do not incorporate the impedance characteristics of the nerve fibers themselves.

The potential distribution in the tissue was calculated using the field simulation for a given stimulus current amplitude. At each time step, the potential distribution along the length of each axon in a simulated population was calculated to determine whether or not the axon's transmembrane potential had depolarized to a point equal to or in excess of a specified threshold value of $25 \mathrm{mV}$. If the threshold potential was exceeded at any point along the axon, the fiber was marked by the program as having fired. The axon equivalent circuit model used in the simulation is consistent with the modeling approach used by Sweeney and colleagues where the conductance of the nerve fiber membrane is assumed to be linear until the threshold potential is reached [14]. We calculate the response of each axon to the excitation current by transforming the axon circuit model and the extracellular potentials into an equivalent intracellularly injected current representation. This representation is more amenable to systematic formulation of the circuit equations and application of transient circuit solution techniques [15]. For a detailed description of the axon equivalent circuit models, the reader is referred to Appendix A.

For these simulations a random population of 60 efferent nerve fibers with a diameter distribution that was consistent with anatomical observations was generated using an inverse interpolation technique [16,17].

Fig. 2 illustrates a population of fiber diameters generated using the same technique as was implemented in the simulation study. This population of sixty fibers was randomly divided into six groups of ten and each group was assigned a depth in the simulated tissue domain at $0.5 \mathrm{~mm}$ increasing intervals starting at a 1 $\mathrm{mm}$ depth. The simulation was carried out with a current pulse density of $1 \mathrm{~A} / \mathrm{m}$ (linear electrode) for stimulus pulse widths of $10 \mu \mathrm{s}, 100 \mu \mathrm{s}, 300 \mu \mathrm{s}, 500 \mu \mathrm{s}, 700 \mu \mathrm{s}$ and $1000 \mu \mathrm{s}$. 

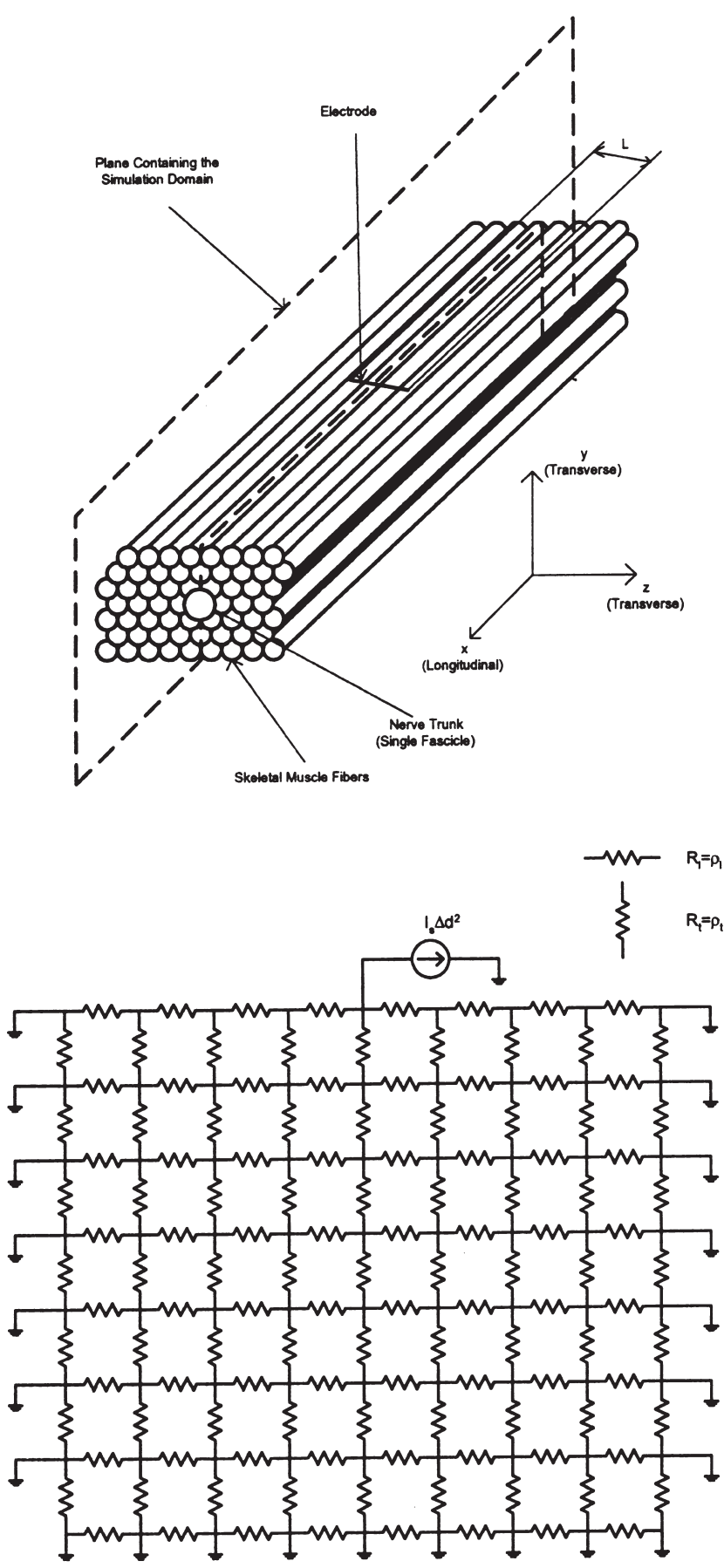

Fig. 1. Idealized representation of the virtual tissue domain used in the field simulations. The vertical and horizontal resistors shown above in the grid are each assigned values consistent with the resistivity observed in the transverse and longitudinal directions, respectively. These values were chosen to be consistent with skeletal muscle tissue such that $R_{\mathrm{t}}=6.75 \Omega \mathrm{m}$ and $R_{\mathrm{l}}=2.4 \Omega \mathrm{m}$. Spacing between the nodes was chosen to be $0.5 \mathrm{~mm}$. In the simulations a $253 \times 252$ node grid was used. The length of the electrode is represented by $L$ and all field related values do not vary in the $z$ direction in the region under the electrode. Fringing effects are not accounted for in this simulation.

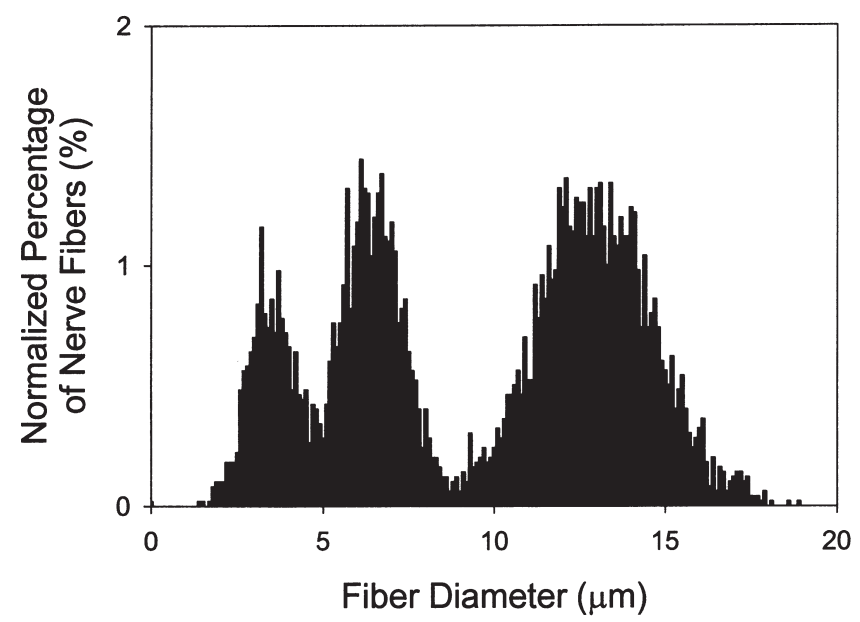

Fig. 2. Efferent peripheral nerve fiber diameter histogram from simulated data. The histogram is drawn from the data plotted for 5000 randomly generated fibers consistent with efferent peripheral nerve distributions presented by Boyd and Davey [18]. Data is plotted as a percentage of the total number of fibers.

\subsection{Experimental studies of recruited motor nerve fiber diameters}

Motor nerve fiber recruitment studies of the thenar muscle on the non dominant hand were carried out on five healthy male subjects, between the ages of 24 and 53 , with no known neurological or neuromuscular pathologies. The stigmatic and reference electrodes were placed over the thenar muscle and proximal phalanx of the thumb as in [2]. A commercial EMG machine (Advantage Medical) using a computer automated motor unit number estimation technique allowed us to extract 20 individual motor unit action potentials and their onset latencies from the sub-maximal M-wave [2]. In this technique, the stimulus amplitude is gradually incremented until 20 motor units have been recruited. Unique motor unit identification is accomplished by pattern recognition applied to the sub-maximal $\mathrm{M}$-wave and the extracted motor unit action potentials. Fig. 3 shows 20 surface recorded motor unit action potentials recorded during one of the experiments. In each study, the median nerve was stimulated on the medial side of the upper arm, proximal to the elbow using $3.5 \mathrm{~cm}$ spacing bipolar electrodes. Independent studies were carried out for three different electrode orientations on each subject. The first electrode orientation was parallel to the length of the upper arm, the second was perpendicular and the third orientation was taken at $45^{\circ}$. In all cases the orientation yielding the maximal $\mathrm{M}$-wave response at the lowest stimulus current was used for the motor unit recruitment studies and motor nerve fiber latency data presented below.

The motor unit recruitment study for each subject was repeated for stimulus current pulse widths of $50 \mu \mathrm{s}$, $100 \mu \mathrm{s}, 200 \mu \mathrm{s}$ and $500 \mu \mathrm{s}$ with the electrode position 


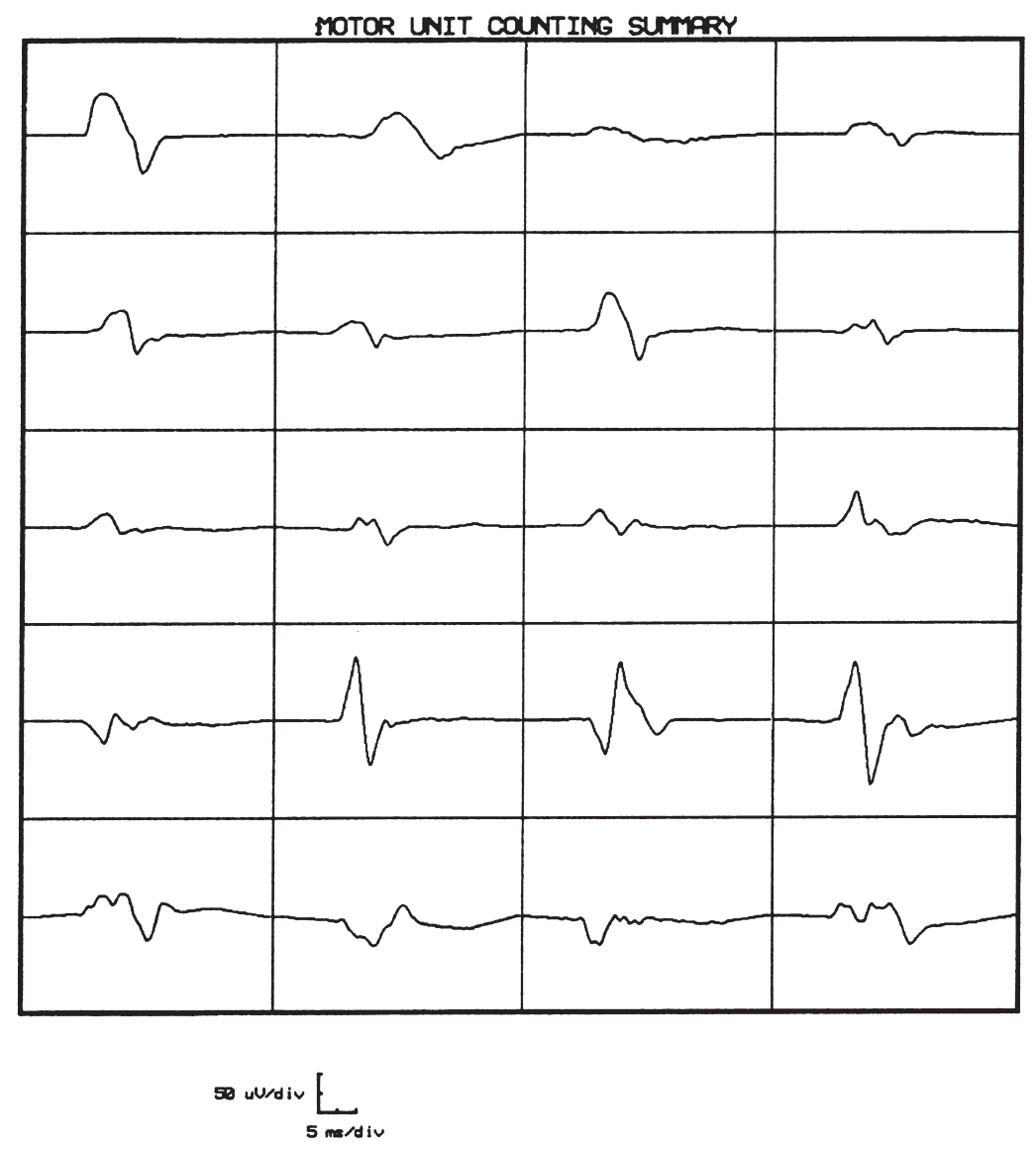

Fig. 3. Surface motor unit action potentials recorded from the thenar muscle for $50 \mu$ s pulse width stimulation of the median nerve proximal to the elbow.

and distance from the nerve unchanged. For all but one of our subjects, the available stimulus current range was sufficient to obtain the full set of twenty different motor unit action potentials at all the stimulus pulse width settings. In one subject, the built in maximal stimulus current amplitude of $100 \mathrm{~mA}$ limited the number of motor units obtainable for the $50 \mu$ s pulse width setting to five. Latency data for each individual motor unit were obtained from measurements of the scaled output shown in Fig. 3. The straight line distance between the stimulus dipole on the upper arm and the recording site on the thenar muscle was recorded for each subject. This information was used in conjunction with the latency data to obtain an estimate of the motor nerve fiber diameter for each individual unit as per Eq. (1) where $d$ is the fiber diameter, $l$ is the estimated straight line distance between the stimulus and the recording electrodes and $t_{\mathrm{d}}$ is the latency associated with each individual motor unit. A constant ratio of fiber conduction velocity to fiber diameter of $c=5.0 \times 10^{6} \mathrm{~s}^{-1}$ was used in estimating the motor nerve fiber diameter of each individual motor unit from the conduction velocity estimates [19].

$$
d=\frac{l}{c t_{\mathrm{d}}}
$$

\section{Results}

\subsection{Simulated motor nerve fiber recruitment patterns}

The average diameter of recruited nerve fibers, as a function of stimulus pulse width, is plotted in Fig. 4. Three separate plots are shown on the graph for increasing distance between the simulated nerve trunk and the stimulus electrodes.

Fig. 4 shows that the average recruited fiber diameter decreases with increasing pulse width. This result is consistent with expectations since larger diameter nerve fibers are recruited more easily than smaller diameter fibers. Stimulus strength versus stimulus duration curves also demonstrate this effect since a given fiber's stimulus threshold decreases with increasing pulse width or 


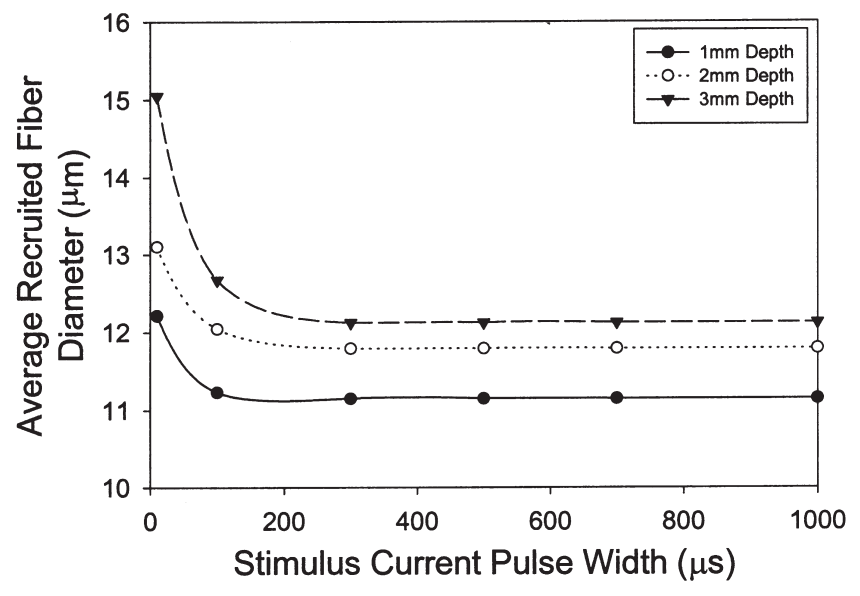

Fig. 4. Plot of simulations of average recruited nerve fiber diameter under conditions of variable stimulus current pulse width.

energy. Hence smaller, higher threshold fibers are increasingly recruited as pulse energy increases. Fig. 4 also shows that the overall average recruited fiber diameter increased as the depth or the distance between the stimulus source and the nerve fibers increased. This result is also consistent with expectations, since as the distance between the nerve fibers and the stimulus source increases, only somewhat larger lower threshold nerve fibers would be recruited.

Fig. 5 shows the average diameter of recruited nerve fibers, normalized to the average calculated for a $10 \mu \mathrm{s}$ pulse. This figure demonstrates the relative effects of changing pulse width, independent of nerve trunk depth in the tissue. As can be seen, the relative effects increase with increasing nerve trunk distance from the electrode. This result is consistent with previous reports [7].

There was also an increase in the absolute range of the recruited fibers as the simulated pulse width was increased from $10 \mu \mathrm{s}$ to $1 \mathrm{~ms}$. This result implies that

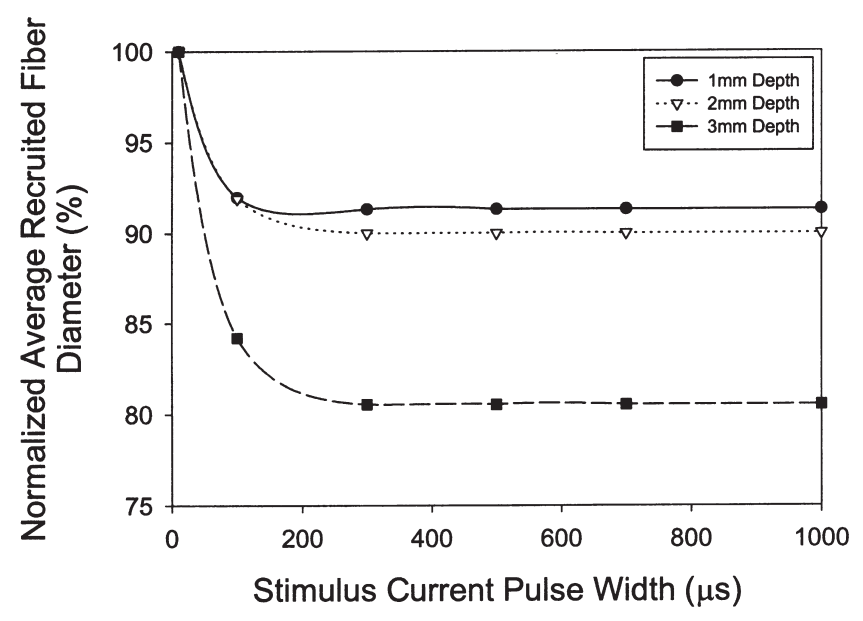

Fig. 5. Plot of simulations of average recruited nerve fiber diameter under conditions of variable stimulus current pulse width. Each curve is normalized to the average recruited fiber diameter for a $10 \mu$ s pulse. for wider stimulus pulse widths, the average recruited fiber diameter decreases and the overall population of recruited fibers increases as well as more fibers of different sizes are excited to threshold.

Fig. 6 illustrates the increase in the absolute range of the recruited fibers as the stimulus pulse width increases from $10 \mu \mathrm{s}$ to $1 \mathrm{~ms}$. The general trend observed is a recruitment of smaller diameter fibers at increasing pulse widths. Due to the fact that the fiber population is distributed across a range of depths starting at $2 \mathrm{~mm}$, some larger fibers at greater depths are recruited as the stimulus current pulse width increases.

\subsection{Experimental motor nerve fiber recruitment studies}

All of the subjects studied exhibited a decrease in average diameter of recruited nerve fibers as stimulus current pulse width increased from $50 \mu$ s to $500 \mu$ s. Fig. 7 shows the normalized average recruited fiber diameter as a function of pulse width. Although Fig. 7 shows the expected negative slope, it is not as consistent as for the simulation results of Fig. 5. As well the relative change in average fiber diameter is not as great, even though the nerve to electrode distance is greater than $3 \mathrm{~mm}$. It should however be noted that the pulse width range for the experimental study was $50 \mu$ s to $500 \mu$ s while the simulation study encompasses a pulse width range of 10 $\mu$ s to $1000 \mu \mathrm{s}$.

All subjects studied exhibited an increase in the range of maximum versus minimum diameter nerve fibers that were recruited at some point as the stimulus current pulse width was increased. The results however do show that this range does not always consistently increase as the stimulus current pulse width increases. A maximum difference between the largest to smallest diameter fibers recruited was observed in the middle range of the stimulus current pulse widths for most subjects. Three of the five subjects that we studied exhibited an overall increased range of recruited fibers when we compared the smallest to largest diameter fiber recruited at $50 \mu \mathrm{s}$ and $500 \mu$ s stimulus pulse widths.

\section{Discussion}

The trends observed in the simulation study are consistent with other studies investigating the differences in stimulus current required to recruit a fiber under conditions of variable stimulus pulse width and distance from the excitation source. The simulations presented in this paper exhibit a small (up to approximately 20\%) decrease in the average recruited nerve fiber diameter when the stimulus pulse width is changed from $10 \mu \mathrm{s}$ to $1000 \mu \mathrm{s}$. As well, the variations in average recruited nerve fiber diameter are greater when the nerve fiber is 
$10 \mu$ s Pulse Width

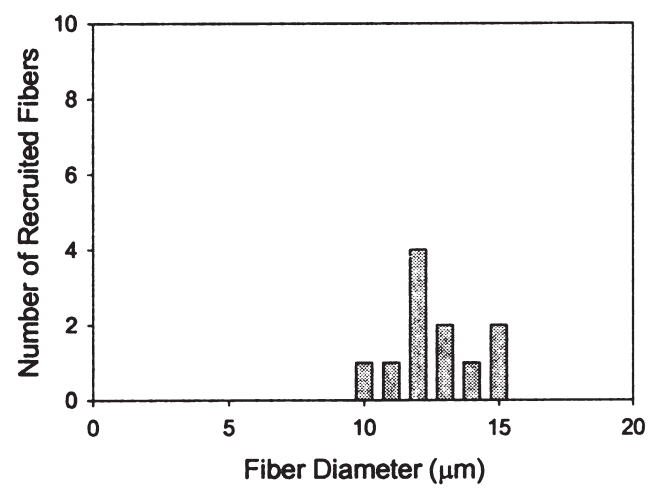

$300 \mu$ s Pulse Width

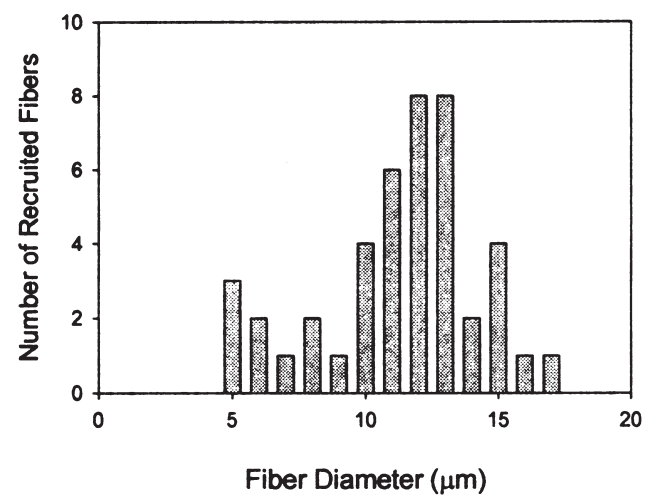

$100 \mu$ s Pulse Width

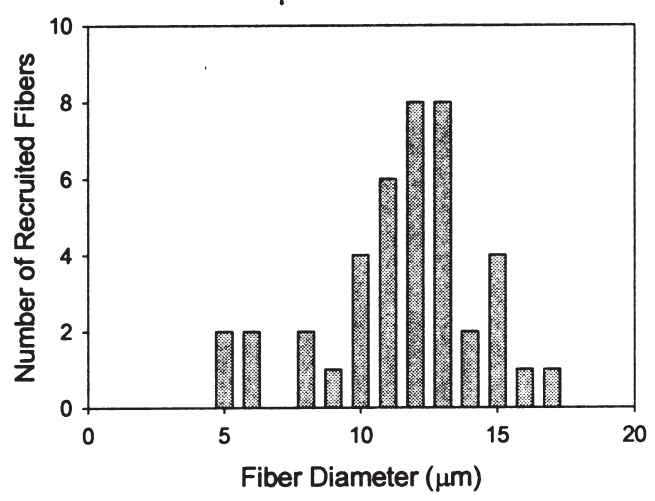

1 ms Pulse Width

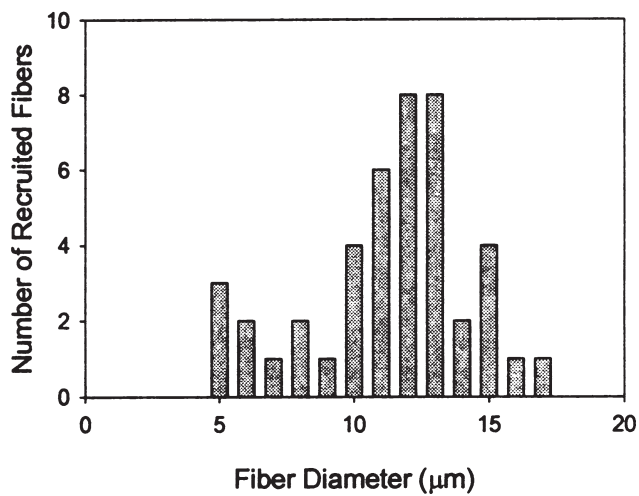

Fig. 6. Histograms of the recruitment order of nerve fibers in the $2 \mathrm{~mm}$ electrode fiber group spacing simulations. As can be seen from the histograms, the distribution or recruited nerve fibers remains the same for pulse widths between $300 \mu \mathrm{s}$ and $1 \mathrm{~ms}$.

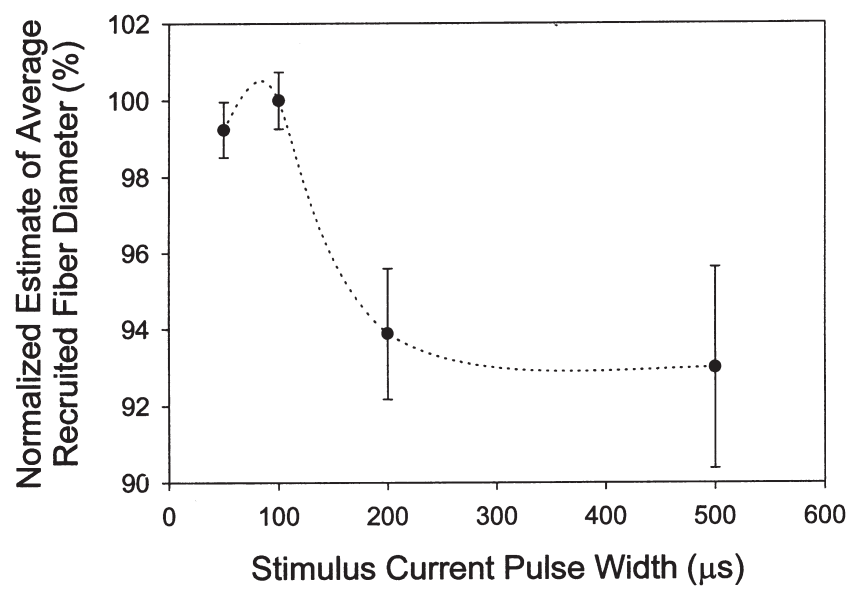

Fig. 7. Plot of the estimate of the average recruited nerve fiber diameter as a function of stimulus current pulse width. These are the averages from data taken from five subjects where each subject's data have been normalized to the maximum average recruited fiber diameter estimate. The average nerve fiber diameter is estimated from data taken from motor unit recruitment studies conducted at different stimulus current pulse widths. Standard error bars are shown for the motor unit estimation study. positioned farther from the stimulus electrodes in the simulated tissue domain. However it must be noted that these results are also dependent on the fiber diameter distribution and relative location of the population of nerve fibers used in these simulations.

The trends observed in the motor unit recruitment studies that were performed on several subjects are consistent with the simulation results, giving some validity to the simulation approach. These results suggest that the nerve fiber, and consequently motor unit size, selectivity that is achievable by varying the stimulus current pulse width within the commonly used clinical range, is not very promising. Only relatively small variations in the average recruited nerve fiber diameter of approximately $1 \mu \mathrm{m}$ were observed in the experimental study, when the stimulus pulse width was increased from 50 $\mu \mathrm{s}$ to $500 \mu \mathrm{s}$. This was considerably less than the approximately $20 \%$ change observed in Fig. 5 at $3 \mathrm{~mm}$ depth in the simulation results, even though the median nerve is probably greater than $3 \mathrm{~mm}$ away from the surface stimulating electrode. However, the majority of the variation observed in the simulations occurs in the range of $10 \mu$ s to $100 \mu \mathrm{s}$. Since the shortest pulse in the experi- 
mental studies was $50 \mu$ s because of machine limitations, the apparent difference between simulation and experimental results is not as significant, since we could not verify the $10 \mu$ s to $50 \mu$ s range.

Although simulation results suggest greater achievable size selectivity at increasing distance between the stimulus source and the nerve fibers, a consequence of greater distance is the necessity to use larger stimulus amplitudes to recruit more fibers when a maximal response is desired. Larger stimulus current amplitudes have the disadvantage of causing greater discomfort to the subjects due to excitation of local sensory fibers. Utilizing narrower stimulus current pulse widths also has the consequence of requiring larger stimulus current amplitudes to recruit the nerve fibers.

The results presented here also provide evidence of the robustness of the McComas motor unit number estimation technique [2] performed with surface stimulus pulse widths that vary across the commonly used clinical range. The results suggest that these motor unit number estimates are relatively independent of the stimulus current pulse width that is used. However, both simulation and experimental results indicate that the range of recruited fiber diameters increases with increasing pulse width. The use of pulse widths greater than $50 \mu$ s could stimulate a more representative sample of motor units. Whether this is true or not requires further investigation.

Although the estimates of nerve fiber diameter based on latency measurements from motor unit recruitment studies demonstrate relatively small variations in the average recruited nerve fiber diameter, it may well be that these variations are marginally greater than demonstrated by this technique. The reason for this hypothesis is the fact that the individual fiber action potentials that sum to form the individual motor unit action potentials may be expected to exhibit a degree of temporal dispersion because of fiber endplate dispersion and different fiber distances to the recording electrode for the same motor unit. The overall effect would be a compression or masking of the observed temporal latencies in the motor unit estimates.

The theoretical prediction of the relatively small variation in motor nerve fiber recruitment patterns coupled with consistent experimental evidence suggests that there is little potential for achieving significant motor nerve fiber recruitment selectivity by varying the stimulus pulse width over the clinically useful range of $50 \mu \mathrm{s}$ to $500 \mu$ s when surface stimulation is used. Our results also suggest that for motor unit number estimation with surface stimulation, the sample of nerve fibers recruited at one electrode location cannot be significantly changed by varying the stimulus current pulse width over the clinically used range. However there is some evidence that a wider range of fiber diameters is recruited at longer pulse widths.

\section{Acknowledgements}

This work was supported by a grant from the Natural Sciences and Engineering Research Council of Canada

\section{Appendix A. Myelinated axon equivalent circuit modeling}

To model the effects of the imposed extracellular field on the myelinated nerve axon a cable equivalent circuit model was used that consists of passive conductances and, in the time domain simulation, passive capacitances. This modeling approach is similar to the circuits used by Bean [13], Sweeney et al. [14] and McNeal [20] (Table 1). There are several assumptions inherent to this modeling approach. In actuality, the transmembrane conductance per unit area of the nerve axon is a non-linear function of the transmembrane potential and time. To simplify analysis, the assumption is made that the conductance of the membrane is linear up until the nerve fiber reaches excitation [13].

Assuming a linear model is a simplification that makes the problem of estimating the firing probabilities of a population of axons computationally tractable. The increase in computational cost associated with non-linear models is significant [21] and it has been acknowledged that the assumption of constant membrane conductances in all nodes is appropriate in computationally intensive situations [14,22].

Fig. 8 illustrates a section of the myelinated nerve axon and the associated electrical equivalent circuit model. At the Nodes of Ranvier there is an exposed section of membrane of approximately $2.5 \mu \mathrm{m}$ in length. Only at these points is the membrane exposed to the extracellular environment. The intervening areas of membrane between the Nodes of Ranvier are insulated by Schwann cells in peripheral nerves, which are assumed to have an extremely high impedance relative

Table 1

Summary of the parameter values and formulas from McNeal [20] used to calculate the equivalent circuit components

\begin{tabular}{lll}
\hline$\rho_{\mathrm{a}}$ & Cytoplasm resistivity & $1.1(\Omega \mathrm{m})$ \\
$g_{\mathrm{m}}$ & Membrane conductance & $304\left(\mathrm{~S} / \mathrm{m}^{2}\right)$ \\
$c_{\mathrm{m}}$ & Membrane capacitance & $0.02\left(\mathrm{~F} / \mathrm{m}^{2}\right)$ \\
$l$ & Node of Ranvier width & $2.5(\mu \mathrm{m})$ \\
$D$ & Fiber diameter & $(\mathrm{m})$ \\
$d$ & Axon diameter & $(\mathrm{m})$ \\
$A$ & Fiber radius & $(\mathrm{m})$ \\
$a$ & Axon radius & $(\mathrm{m})$ \\
$a / A$ & Ratio of axon to fiber radius & 0.7 \\
$K$ & Nodes of Ranvier spacing & $100 \times D(\mathrm{~m})$ \\
$R_{\mathrm{c}}$ & Equivalent axoplasm resistance & $\left(\rho_{\mathrm{a}} K\right) /\left(\pi \mathrm{a}^{2}\right)(\Omega)$ \\
$R_{\mathrm{m}}$ & Equivalent membrane resistance & $\left(2 \pi g_{\mathrm{m}} a l\right)^{-1}(\Omega)$ \\
$C_{\mathrm{m}}$ & Equivalent membrane & $\left(2 \pi c_{\mathrm{m}} a l\right)(\mathrm{F})$ \\
& capacitance & \\
\hline
\end{tabular}




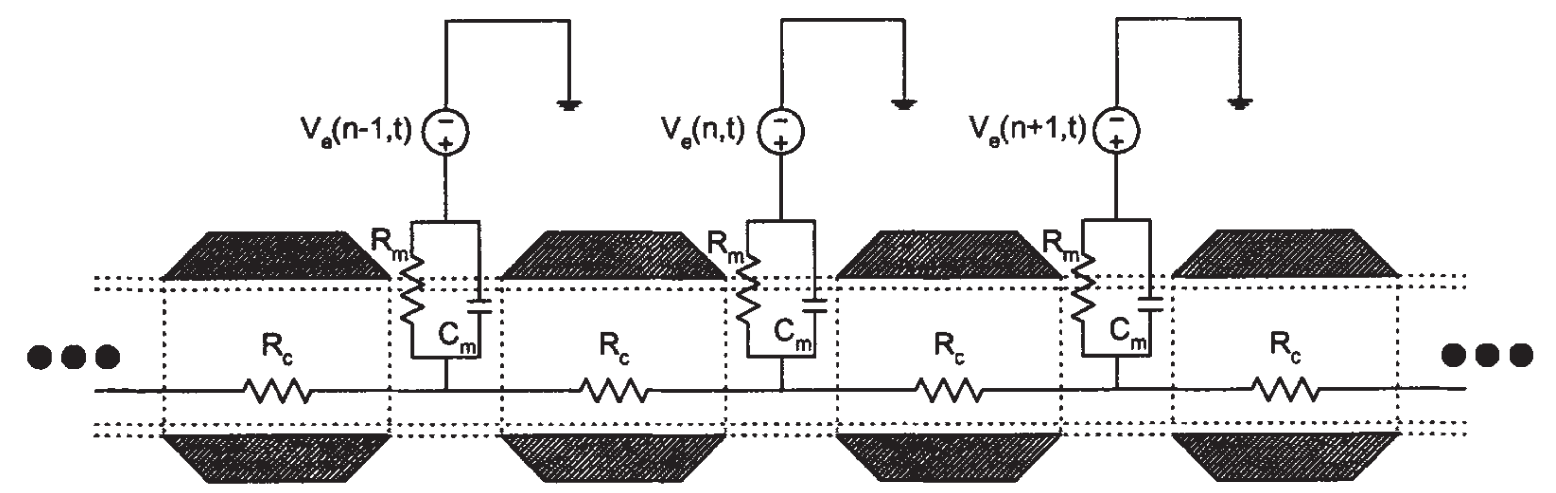

Fig. 8. Equivalent circuit model of a section of myelinated axon. The circuit is superimposed on the axial cross section of the fiber in an attempt to relate the equivalent circuit components to the physical structure of the axon. $R_{\mathrm{c}}$ and $R_{\mathrm{m}}$ represent the resistance of the cytoplasm and the exposed section of membrane, respectively. The parameter $C_{\mathrm{m}}$ represents the capacitance associated with the exposed section of the membrane. Extracellular potentials at the Nodes of Ranvier are represented by $V_{\mathrm{e}}$ where $n$ denotes the specific Node of Ranvier. The membrane resting potential sources $V_{\mathrm{r}}$ are not shown in this figure.

to the bare membrane. Consequently, the assumption is made in virtually all models presented in the literature that the Schwann cells are effectively non-conducting.

Kirchoff's current law can be invoked at the intracellular nodes to write a differential equation for the equivalent circuit model shown in Eq. (A.1) where $V_{\mathrm{m}}$ is the transmembrane potential. The transmembrane potential can also be written as $V_{\mathrm{m}}=V_{\mathrm{i}}-V_{\mathrm{e}}-V_{\mathrm{r}}$ where $V_{\mathrm{i}}$ is the intracellular potential at each node and $V_{\mathrm{r}}$ is the membrane resting potential $[12,14]$.

$$
\begin{aligned}
& C_{\mathrm{m}} \frac{\mathrm{d} V_{\mathrm{m}}(n, t)}{\mathrm{d} t}+G_{\mathrm{m}} V_{\mathrm{m}}(n, t)+G_{\mathrm{c}}\left[V_{\mathrm{e}}(n, t)+V_{\mathrm{m}}(n, t)\right] \\
& \quad-G_{\mathrm{c}}\left[V_{\mathrm{e}}(n-1, t)+V_{\mathrm{m}}(n-1, t)\right]+G_{\mathrm{c}}\left[V_{\mathrm{e}}(n, t)+V_{\mathrm{m}}(n, t)\right]
\end{aligned}
$$

$$
\begin{gathered}
-G_{\mathrm{c}}\left[V_{\mathrm{e}}(n+1, t)+V_{\mathrm{m}}(n+1, t)\right]=0 \\
C_{\mathrm{m}} \frac{\mathrm{d} V_{\mathrm{m}}(n, t)}{\mathrm{d} t}+G_{\mathrm{m}} V_{\mathrm{m}}(n, t)-G_{\mathrm{c}}\left\{V_{\mathrm{m}}(n-1, t)\right. \\
\left.-2 V_{\mathrm{m}}(n, t)+V_{\mathrm{m}}(n+1, t)\right\}=G_{\mathrm{c}}\left\{V_{\mathrm{e}}(n-1, t)\right. \\
\left.-2 V_{\mathrm{e}}(n, t)+V_{\mathrm{e}}(n+1, t)\right\}
\end{gathered}
$$

In the differential equations, $G_{\mathrm{c}}$ and $G_{\mathrm{m}}$ are the conductances or the inverse of the resistors $R_{\mathrm{c}}$ and $R_{\mathrm{m}}$, respectively, shown in Figs. 8 and 9. It should also be noted that the terms representing the extracellular potentials and the excitation sources in Eqs. (A.1) and (A.2)

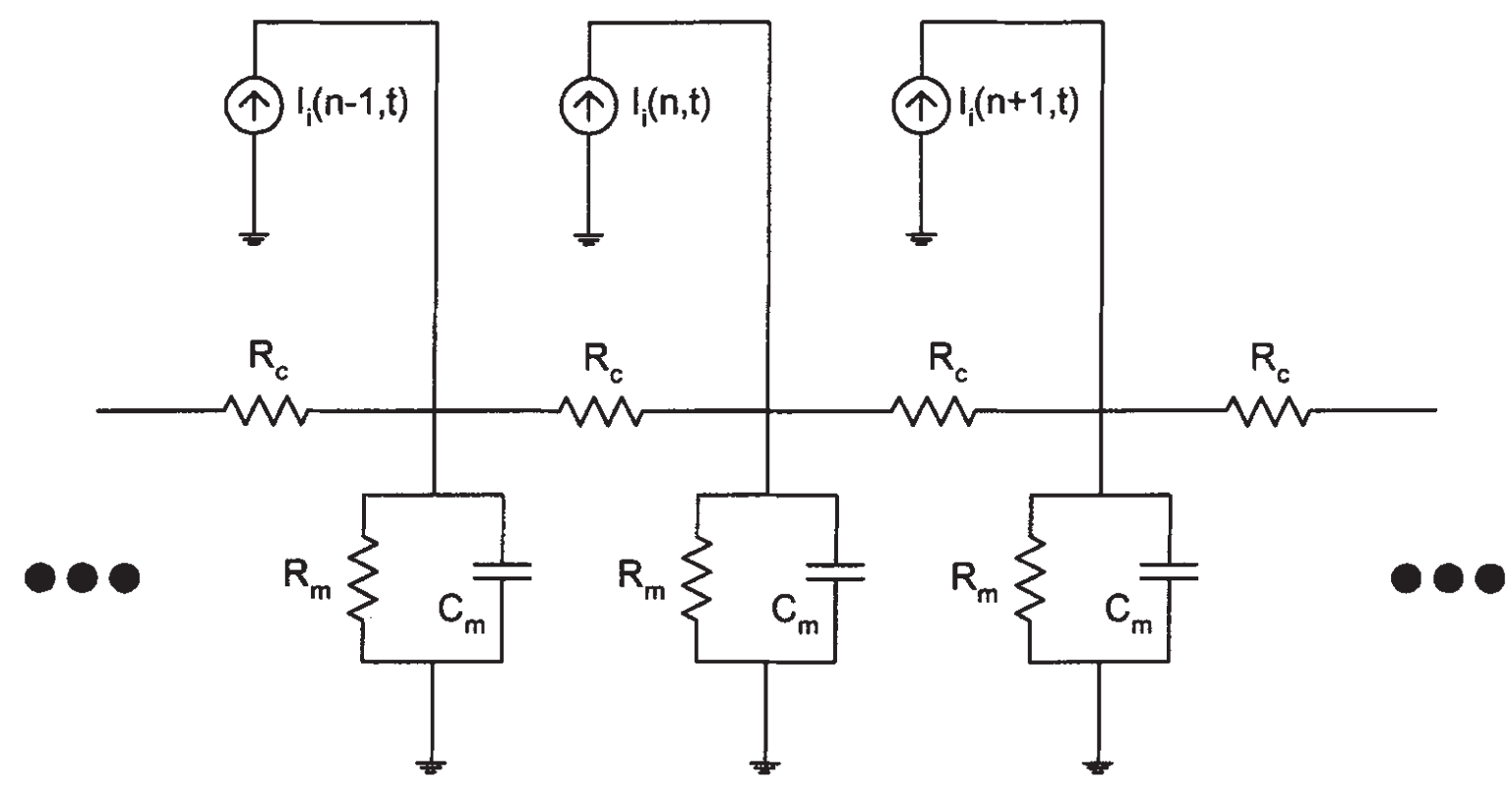

Fig. 9. Intracellularly injected current equivalent circuit model of a myelinated axon. The circuit models of Figs. 8 and 9 are electrically equivalent provided that the current sources in Fig. 9 are specifically chosen. Circuit parameters $R_{\mathrm{m}}, C_{\mathrm{m}}$ and $R_{\mathrm{c}}$ are defined the same way as in Fig. 8. The intracellularly injected current sources labeled $I_{\mathrm{i}}$ are specific to the Node of Ranvier as indexed by $n$. The membrane resting potential sources $V_{\mathrm{r}}$ are not shown in this figure. 
are functions of both the position of the different Nodes of Ranvier, as indexed through the parameter $n$, and time.

From a theoretical perspective, it can be shown that the potentials applied to the outside of the fiber are equivalent to a set of current sources applied intracellularly if these current sources are defined in a specific way in terms of the extracellular potentials. The equivalent circuit with intracellularly injected current sources is shown in Fig. 9.

The differential equation for the intracellularly injected current equivalent circuit model is shown in Eq. (A.2).

$$
\begin{gathered}
C_{\mathrm{m}} \frac{\mathrm{d} V_{\mathrm{m}}(n, t)}{\mathrm{d} t}+G_{\mathrm{m}} V_{\mathrm{m}}(n, t)-G_{\mathrm{c}}\left\{V_{\mathrm{m}}(n-1, t)\right. \\
\left.-2 V_{\mathrm{m}}(n, t)+V_{\mathrm{m}}(n+1, t)\right\}=I_{\mathrm{i}}(n, t)
\end{gathered}
$$

In order for the two models to be equivalent, the intracellularly injected current sources $I_{\mathrm{i}}(n, t)$ must be chosen such that they are equivalent to the right hand term of Eq. (A.1) as per Eq. (A.3).

$$
I_{\mathrm{i}}(n, t)=G_{\mathrm{c}}\left\{V_{\mathrm{e}}(n-1, t)-2 V_{\mathrm{e}}(n, t)+V_{\mathrm{e}}(n+1, t)\right\}
$$

It is a relatively straightforward task to compute the values of the equivalent circuit components $C_{\mathrm{m}}, R_{\mathrm{m}}$ and $R_{\mathrm{c}}$ since they are based on the electrical properties of the axon membrane and the cytoplasm as well as the idealized geometry that has been assumed for the nerve axon.

\section{References}

[1] McComas AJ, Fawcett PRW, Campbell MJ, Sica REP. Electrophysiological estimation of the number of motor units within a human muscle. Journal of Neurology Neurosurgery and Psychiatry 1971;34:121-31.

[2] Galea V, De Bruin H, Cavasin R, McComas AJ. The numbers and relative sizes of motor units estimated by computer. Muscle and Nerve 1991;14:1123-30.

[3] Erlanger J, Gasser HS. Electrical Signs of Nervous Activity. Philadelphia: University of Pennsylvania Press, 1968.

[4] Gorman PH, Mortimer JT. The effect of stimulus parameters on the recruitment characteristics of direct nerve stimulation. IEEE Transactions on Biomedical Engineering 1983;BME-30:407-14.

[5] Koole P, Holsheimer J, Struijk JJ, Verloop AJ. Recruitment characteristics of nerve fascicles stimulated by a multigroove electrode. IEEE Transactions on Rehabilitation Engineering 1997:5:40-50.

[6] Meier JH, Rutten WLC, Zoutman AE, Boom HBK, Bergveld P. Simulation of multipolar fiber selective neural stimulation using intrafascicular electrodes. IEEE Transactions on Biomedical Engineering 1992;39:122-34.

[7] Grill WM, Mortimer JT. Stimulus waveforms for selective neural stimulation. IEEE Engineering in Medicine and Biology 1995; 14:375-85.

[8] Grill WM, Mortimer JT. The effect of stimulus pulse duration on selectivity of neural stimulation. IEEE Transactions on Biomedical Engineering 1996;43:161-6.

[9] Solomonow M, Eldred E, Lyman J, Foster J. Control of muscle contractile force through indirect high-frequency stimulation. American Journal of Physical Medicine 1983;62:71-82.

[10] Gielen FLH, Cruts HEP, Albers BA, Boon KL, Wallinga-De Jonge W, Boom HBK. Model of electrical conductivity of skeletal muscle based on tissue structure. Medical and Biological Engineering and Computing 1986;24:34-40.

[11] Gielen FLH, Wallinga-De Jonge W, Boon KL. Electrical conductivity of skeletal muscle tissue: experimental results from different muscles in vivo. Medical and Biological Engineering and Computing 1984;22:569-77.

[12] Warman EN, Grill WM, Durand D. Modeling the effects of electric fields on nerve fibers: determination of excitation thresholds. IEEE Transactions on Biomedical Engineering 1992;39:1244-54.

[13] Bean CP. A theory of microstimulation of myelinated fibres. Journal of Physiology 1974;243:514-22.

[14] Sweeney JD, Ksienski DA, Mortimer JT. A nerve cuff technique for selective excitation of peripheral nerve trunk regions. IEEE Transactions on Biomedical Engineering 1990;37:706-15.

[15] Fidler JK, Nightingale C. Computer Aided Circuit Design. Don Mills: Nelson, 1978.

[16] Szlavik RB, de Bruin H. Simulating the distribution of axon size in nerves. Proceedings of the 23rd Canadian Medical and Biological Engineering Conference, 1997:168-169.

[17] Jeruchim MC, Balaban P, Shanmugan KS. Simulation of Communication Systems. New York: Plenum Press, 1994.

[18] Boyd IA, Davey MR. Composition of Peripheral Nerves. Edinburgh: E. and S. Livingstone Ltd, 1968.

[19] Schoonhoven R, Stegeman DF. Models and analysis of compound nerve action potentials. Critical Reviews in Biomedical Engineering 1991;19:47-111.

[20] McNeal DR. Analysis of a model for excitation of myelinated nerve. IEEE Transactions on Biomedical Engineering 1976;BME-23:329-37.

[21] Teicher DA, McNeal DR. Comparison of a dynamic and steadystate model for determining nerve fiber threshold. IEEE Transactions on Biomedical Engineering 1978;BME-25:105-7.

[22] Rattay F. Electrical Nerve Stimulation. New York: Springer-Verlag, 1990. 